detected in the case of one endopeptidase clone, although it did not affect the primary sequence of the protein. This result is not surprising, considering Taq DNA polymerase was used. To reduce the risk of unwanted mutations, a high fidelity polymerase should be used. In the case of longer sequences, the generated clone should be sequence-verified for further studies.

Because of the simplicity and costeffectiveness of the present protocol, we suggest this approach for studies in which numerous small to medium sized genes are to be mutated. The procedure avoids the purchase or creation of cDNA clones and results in high efficiency generation of the desired mutant.

\section{REFERENCES}

1.Allemandou, F., J. Nussberger, H.R. Brunner, and N. Brakch. 2003. Rapid site-directed mutagenesis using two-PCR-generated DNA fragments reproducing the plasmid template. J. Biomed. Biotechnol. 3:202-207.

2.Chen, X., W. Liu, I. Quinto, and G. Scala. 1997. High efficiency of site-directed mutagenesis mediated by a single PCR product. Nucleic Acids Res. 3:682-684.

3.Nagy, Z.B., F. Felföldi, L. Tamás, and L.G. Puskás. 2004. A one-tube, two-step polymerase chain reaction-based site-directed mutagenesis method with simple identification of the mutated product. Anal. Biochem. 324:301303.

4.Sarkar, G. and S.S. Sommer. 1990. The megaprimer method of site-directed mutagenesis. BioTechniques 4:404-407.

5.Steinberg, R.A. and K.B. Gorman. 1994. A high-yield for site-directed mutagenesis using polymerase chain reaction and three primers. Anal. Biochem. 219:155-157.

6.Urban, A., S. Neukirchen, and K.E. Jaeger. 1997. A rapid and efficient method for sitedirected mutagenesis using one-step overlap extension PCR. Nucleic Acids Res. 11:22272228.

7.Puskás, L.G., Á. Zvara, L. Hackler, Jr., T. Micsik, and P. van Hummelen. 2002. Production of bulk amounts of universal RNA for DNA microarrays. BioTechniques 33:898-904.

Received 19 January 2004; accepted 23 February 2004.

Address correspondence to László G. Puskás, Laboratory of Functional Genomics, Biological Research Centre, Hungarian Academy of Sciences, P.O. Box 521, Szeged, H-6701, Hungary. e-mail: pusi@nucleus.szbk.u-szeged.hu

\title{
Improved siRNA-mediated silencing in refractory adherent cell lines by detachment and transfection in suspension
}

\author{
Mohammed Amarzguioui \\ The Biotechnology Centre of Oslo, University of Oslo, Oslo, Norway
}

BioTechniques 36:766-770 (May 2004)

Cationic liposome-based agents (1) are commonly used for cellular delivery (transfection) of oligonucleotides such as short interfering RNA (siRNA) (2). When targeting endogenous gene expression, transfection efficiency is a critical parameter that may vary substantially depending on a combination of cell line and transfection agent, thereby necessitating a time-consuming and expensive optimization of transfection conditions (http://www.molecula. com/new/transfection_optimization. html). It has been previously reported that the human keratinocyte cell line $\mathrm{HaCaT}$ is effectively transfected with an siRNA of optimal activity (hTF167i), which consistently depleted the endogenous target mRNA expression by $90 \%$ (3), using the cationic liposome-based agent Lipofectamine ${ }^{\mathrm{TM}} 2000$ (Invitrogen, Carlsbad, CA, USA). This siRNA proved to be substantially less effective in the human melanoma cell line LOX (4) under a range of transfection conditions, using either Lipofectamine 2000 or the lipid formulation RNAiFect ${ }^{\mathrm{TM}}$ (Qiagen, Hilden, Germany). This suggested that the LOX cells are relatively refractory to transfection with standard methods. Instead of investigating other transfection agents in an attempt to find one that works well with this cell line, alternate methods of utilizing the available agents were considered.

Detachment and co-incubation of cells with complexes while in suspension was considered a possible way of improving transfection efficiency. For this modified transfection protocol, complexes were prepared as usual in $20 \%$ of the final transfection volume (3). Briefly, siRNA was diluted in serum-free medium (1/10 of the final transfection volume) and mixed with an equal volume of medium-diluted liposomes according to the manufacturer's instructions. Cells were prepared for transfection as follows. They were washed twice with phosphate-buffered saline (PBS) and detached by trypsinization. Trypsin was inactivated by the addition of a $1.5 \mathrm{~mL}$ serum $/ 75 \mathrm{~cm}^{2}$ tissue flask of cells. The cell/serum suspension was diluted 10 -fold with PBS and subjected to centrifugation at $400-500 \times g$ for $5 \mathrm{~min}$. The supernatant was removed, and the cells were resuspended in PBS. Following a new centrifugation step and removal of the supernatant, the cells were resuspended in serum-free medium and counted. The cell suspension was adjusted to the appropriate density (typically $0.8-1.0$ $\times 10^{6}$ cells $/ \mathrm{mL}$, see below) and mixed with complexes by careful pipeting before seeding into wells or dishes. Cells were then incubated for $5 \mathrm{~h}$ before replacement of medium. Twice the number of cells normally seeded for nextday transfection of adherent cells were used when performing transfections in suspension (typically $6-8 \times 10^{5}$ cells in $1.0 \mathrm{~mL} / 36-\mathrm{mm}$ well). This was done to compensate for cell expansion from time of seeding to transfection when transfecting adherent cells and for the projected loss of cells that had not attached properly at the time of medium replacement when performing transfections in suspension.

Transfection of LOX cells adherently with hTF167i siRNA under a range of different transfection conditions resulted in knockdown of endogenous target mRNA in the ranges of $51 \%-57 \%$ and $48 \%-57 \%$ (Figure 1, right panel) for the respective transfection agents 
Lipofectamine 2000 and RNAiFect. However, when the cells were transfected in suspension, silencing of gene expression improved to $82 \%$ and $76 \%$ for Lipofectamine 2000 and RNAiFect, respectively (Figure 1, left panel). In a follow-up experiment, although overall levels of silencing were generally reduced, similar relative improvements in silencing were seen (data not shown). After these encouraging observations, the applicability of the above methodology to other combinations of siRNA and difficult-to-transfect cell lines was investigated. An siRNA against murine Tissue Factor (mTF), mTF223i, depletes expression of $\mathrm{mTF}$ in the murine B16 melanoma cell line by $80 \%$ (M. Amarzguioui, Q. Peng, T. Holen, V. Vasovic, E. Babaie, J.M. Nesland, and H. Prydz, unpublished data). In another melanoma cell line, K1735-M4 $(5,6)$, no more than $50 \%$ knockdown was obtained under the same transfection conditions. Experiments were undertaken in which two different active mTF siRNA were evaluated for silencing efficiency when used to transfect K1735M4 cells adherently or in suspension, with either Lipofectamine 2000 or
RNAiFect. Transfection of cells in suspension substantially improved silencing for all combinations of siRNA and transfection agents (Figure 2). For mTF223i, transfection of adherent cells with Lipofectamine 2000 and RNAiFect resulted in (mean \pm SD) $44 \% \pm 9$ $(n=3)$ and $59 \% \pm 8 \%(n=3)$ depletion of mTF mRNA, respectively. The corresponding knockdown levels for cells transfected in suspension with identically prepared complexes were (mean $\pm \mathrm{SD}) 81 \% \pm 3(n=3)$ and $84 \% \pm 3(n$ $=3$ ). This represents a substantial improvement in the degree of silencing, which is likely to be of critical importance for many functional studies.

The silencing efficiencies achieved in more permissive (i.e., easier to transfect) cell lines by either the standard or the modified protocol, using the transfection agent Lipofectamine 2000, were subsequently compared. In MCF-7 cells, the level of silencing achieved by two highly effective siRNAs (wwox1 and wwox2) targeting WWOX mRNA was tested in parallel with both transfection protocols. In the first experiment, transfection in suspension improved the level of knockdown from $75 \%$ to $82 \%$

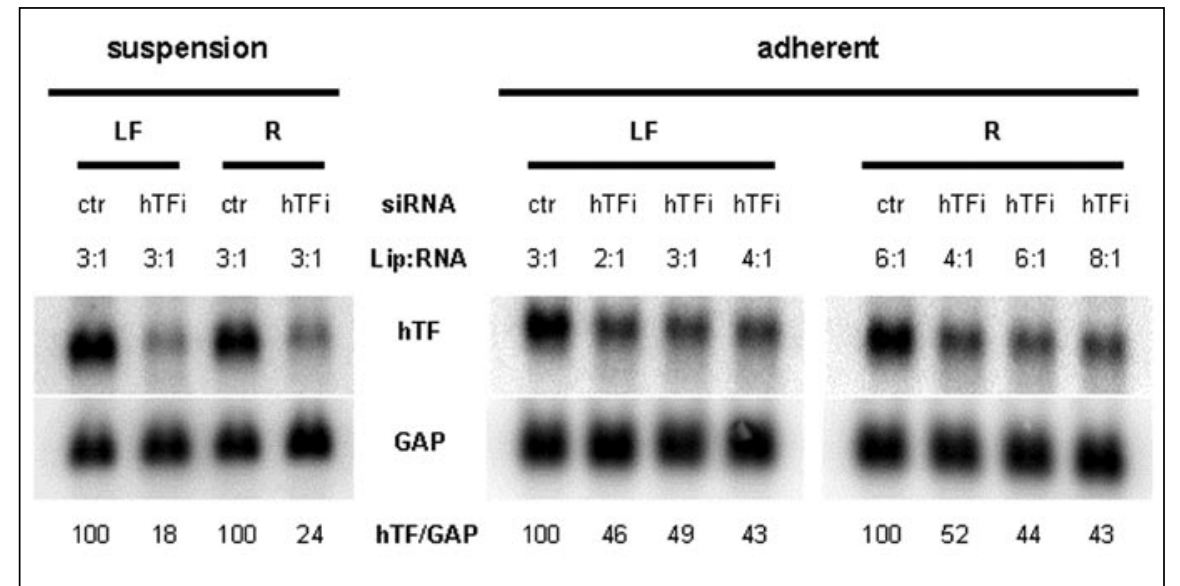

Figure 1. Northern blot analyses comparing short interfering RNA (siRNA)-mediated silencing of human Tissue Factor (hTF) mRNA expression in LOX cells under various transfection conditions. Data from a representative of two independent experiments are shown. Cells were transfected in 6-cm dishes either adherently (right panel) at 40\%-50\% confluency (cells plated at $7 \times 10^{5} /$ dish $18 \mathrm{~h}$ before transfection) or in suspension (left panel) $\left(1.5 \times 10^{6} / \mathrm{dish}\right)$ with $2.0 \mathrm{~mL} 100 \mathrm{nM}$ siRNA complexed with either Lipofectamine 2000 (LF) or RNAiFect (R). ctr, mismatched control siRNA (mTF223i) targeting murine Tissue Factor (mTF); hTFi, active hTF-specific siRNA (hTF167i). Complexes had liposomes to RNA ratios (Lip:RNA, v/w, $\mu \mathrm{L}$ liposome to $\mu \mathrm{g}$ RNA) as indicated. Cells were harvested $48 \mathrm{~h}$ posttransfection for mRNA isolation using Dynabeads ${ }^{\circledR}$ Oligo(dT) ${ }_{25}$ (Dynal Biotech, Oslo, Norway). Northern blot analyses were hybridized with full-length cDNA probes for hTF and GAPDH in PerfectHyb ${ }^{\mathrm{TM}}$ Plus Hybridization Buffer (Sigma, St. Louis, MO, USA) according to the manufacturer's instructions. GAPDH expression served as loading and normalization control. Hybridized membranes were scanned, and images were analyzed using the Molecular Dynamics Storm ${ }^{\circledR} 860$ scanner and ImageQuant ${ }^{\mathrm{TM}} 5.1$ software (both from Amersham Biosciences, Little Chalfont, Buckinghamshire, UK). GAP, GAPDH. 


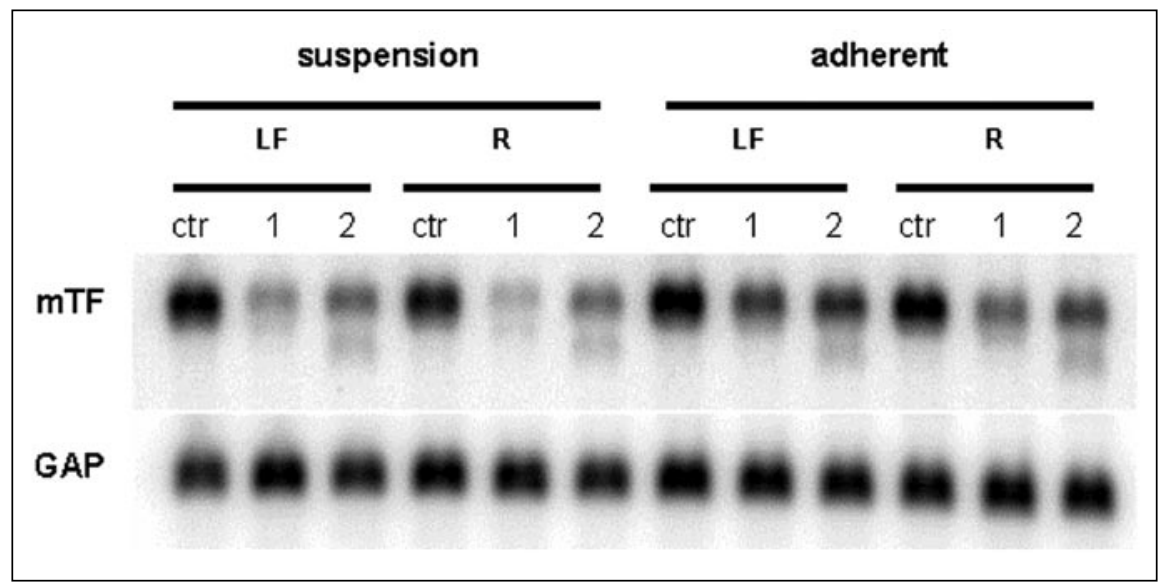

Figure 2. Northern blot analyses comparing short interfering RNA (siRNA)-mediated silencing of murine Tissue Factor (mTF) mRNA expression in K1735-M4 cells under various transfection conditions. Data from a representative of three independent experiments are shown. Ctr, mismatched control siRNA (hTF167i) targeting human Tissue Factor (hTF). Lanes 1 and 2, active mTF-specific siRNA (mTF223i and mTF321i, respectively). Complexes have optimized ratios (v/w) of liposomes to RNA of 3:1 for Lipofectamine 2000 (LF) and 6:1 for RNAiFect (R). Northern blot analyses were performed as described for Figure 1. GAP, GAPDH.

for wwox 1 and from $71 \%$ to $80 \%$ for wwox2. Similar improvements were seen for both siRNA in a follow-up experiment (from $80 \%$ to $88 \%$ for wwox 1 and from $85 \%$ to $89 \%$ for wwox 2 ). Thus, although the differences were relatively small due to the high initial silencing efficiency, they were consistent across four separate side-by-side comparisons, with an average percentage point increase in silencing of $7.0 \%$ $\pm 2.2(n=4)$. The above data, in addition to preliminary data from two other human cell lines, suggest that performing transfections in suspension results in slightly improved results even for easily transfectable cell lines. Transfection of $\mathrm{HaCaT}$ cells in suspension did not result in significant improvement of silencing achieved with siRNA of low intrinsic activity. This further supports the notion that transfection in suspension increases the number of functionally transfected (i.e., silenced) cells, which in $\mathrm{HaCaT}$ cells is close to $100 \%$ even with the standard protocol.

The reasons for the improvements in silencing observed with the modified protocol reported here are not clear. One possibility is that a more favorable cell surface environment for attachment of complexes is generated following partial removal of surface proteins by trypsinization. If this were the case, improvements should also be expected for cells normally cultured in suspension if they were to be subjected to a trypsinization step prior to mixing with the liposome complexes. However, Lipofectamine 2000-mediated transfection of Jurkat cells failed to demonstrate trypsin treatment step in the preparation of cells for transfection (data not shown). Trypsinization may improve transfection efficiency of adherent cells through increased surface availability. It is also possible that the critical factor is the presence of complexes on the cells at the time when they undergo morphological changes associated with attachment to the surface of the culture plates or dishes, which may render them more easily transfectable.

In conclusion, this report has demonstrated that silencing of endogenous gene expression in cells that are normally resistant to transfection with two different commonly used agents can be improved substantially by mixing the complexes with cells in suspension and allowing the cells to attach in the presence of the complexes. This strategy has been shown to improve silencing for all combinations of refractory cell line, siR$\mathrm{NA}$, and transfection agent tested. While the method has only been evaluated for the cationic liposome-based agent Lipofectamine 2000 and the lipid formulation RNAiFect, the applicability of the method is not expected to be restricted to the reagents mentioned above. any effect of incorporating a 5-min

\section{ACKNOWLEDGMENTS}

This work was supported by grants from the Norwegian Cancer Society, Health and Rehabilitation, and the Research Council of Norway (RCN) to Hans Prydz. M. Amarzguioui is a postdoctoral research fellow at $R C N$.

\section{REFERENCES}

1.Felgner, P.L. and G.M. Ringold. 1987. Cationic liposome-mediated transfection. Nature 337:387-388.

2.Elbashir, S.M., J. Harborth, W. Lendeckel, A. Yalcin, K. Weber, and T. Tuschl. 2001 Duplexes of 21-nucleotide RNAs mediate RNA interference in cultured mammalian cells. Nature 411:494-498

3.Holen, T., M. Amarzguioui, M.T. Wiiger, E. Babaie, and H. Prydz. 2002. Positional effects of short interfering RNAs targeting the human coagulation trigger Tissue Factor. Nucleic Acids Res. 30:1757-1766.

4.Fodstad, O., S. Aamdal, M. McMenamin, J.M. Nesland, and A. Pihl. 1988. A new experimental metastasis model in athymic nude mice, the human malignant melanoma LOX. Int. J. Cancer 41:442-449.

5.Fidler, I.J., E. Gruys, M.A. Cifone, Z. Barnes, and C. Bucana. 1983. Demonstration of multiple phenotypic diversity in a murine melanoma of recent origin. J. Natl. Cancer Inst. 67:947-956.

6.Voigtlander, C., A. Rand, S.L. Liu, T.J. Wilson, M.R. Pittelkow, M.J. Getz, and R.J. Kelm, Jr. 2002. Suppression of tissue factor expression, cofactor activity, and metastatic potential of murine melanoma cells by the $\mathrm{N}$ terminal domain of adenovirus E1A $12 \mathrm{~S}$ protein. J. Cell. Biochem. 85:54-71.

Received 2 March 2004; accepted 29 March 2004.

Address correspondence to Mohammed Amarzguioui, Department of Molecular Biology, Beckman Research Institute of the City of Hope, Duarte, CA 91010, USA.e-mail: moamarzg@biotek.uio.no or mamarzguio@coh.org 